\section{Experimental Verification at High Pressure of the Relationship between Compression, Density and Sonic Velocity}

THE equation relating density, compressibility and sonic velocity can be expressed as

$$
C=\left(V K_{s}\right)^{1 / 2}
$$

where $C$ is the velocity of sonic wave propagation, $V$ is the volume of unit mass, $K_{s}$ is the isentropic bulk modulus $=-V(\partial P / \partial V)_{s}:$ and $s$ is entropy.

Although the equation is often treated as if it were a thermodynamic relation, various writers, notably Richards ${ }^{1}$ and Rowlinson ${ }^{2}$, have pointed out that this is not so, but that it requires experimental verification, which is available for liquids at atmospheric pressure only. Because the equation is used industrially to derive compressibilities of hydraulic fluids at high pressures, such verification is of practical importance. Its absence is no doubt a result of the difficulty of measuring isentropic compressibility at high pressures.

These difficulties have now been overcome, and accurate direct measurements of $K_{8}$ have been made at pressures up to $\mathbf{1 . 2} \mathrm{kbar}$ for what I believe to be the first time. I have already described the apparatus ${ }^{3}$, but recent improvements to the operating technique have enabled me to obtain accuracies of the order of 1 per cent at high pressures. Measurements are made by forcing a piston rapidly into a sealed cylinder filled with the test liquid, and immediately withdrawing the piston at the same speed. An automatic testing machine is used to apply the load, so that curves of load against piston displacement are automatically drawn for both the loading and unloading operations. A seal has been developed which is practically friction free, and thermal losses are negligible; the combined effects of friction and thermal loss are such as to displace the unloading curve from the loading curve by less than 1 per cent. Consequently a mean line between the loading and unloading curves can be regarded as a true isentropic curve.

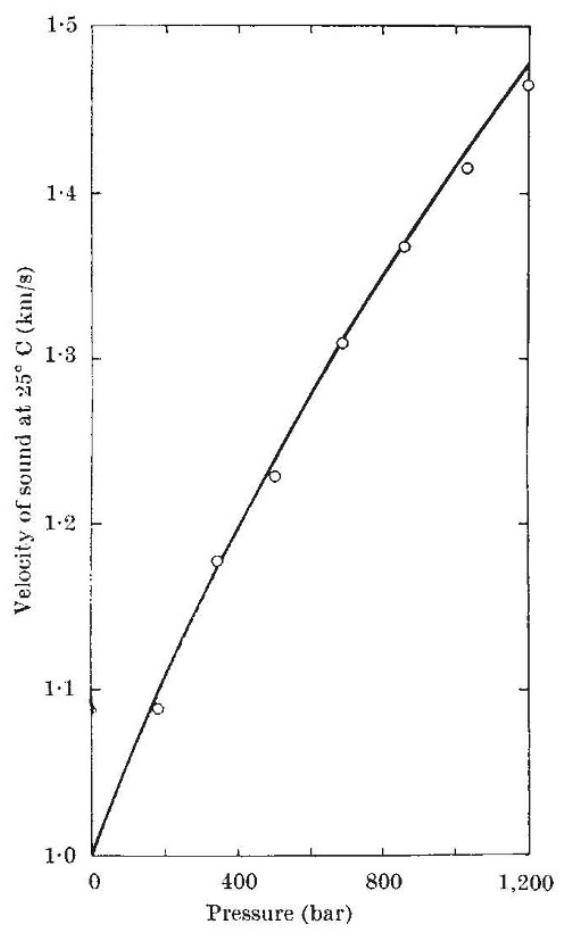

Fig. 1. Comparison between measured and derived values of sonic velocity in dimethyl silicone fluid. —-, Mean line derived from direct measurements by Yazgan; $O$, valucs calculated from compressibility measurements.
A dimethyl silicone oil (viscosity 12,500 centistokes) was chosen for the experiment, because I thought that a viscous polymeric liquid was most likely to exhibit any anomalous effects that might exist. Sonic velocities were measured by Yazgan ${ }^{4}$ on separate portions of the same sample of fluid at frequencies of 10 and $30 \mathrm{MHz}$. No dispersion was detected. The curve of sonic velocity against pressure obtained by Yazgan at $25^{\circ} \mathrm{C}$ is shown in Fig. 1 .

Isothermal and isentropic compressibilities at $25^{\circ} \mathrm{C}$ were measured at seven pressures from 200 to 1,200 bar inclusive. The isothermal results were used to derive $V$ at elevated pressures. Values of $K_{s}$ were obtained by measuring the change in isentropic volume from 100 bar below to 100 bar above the test pressure, during which time the temperature rose from $21.7^{\circ}$ to $28 \cdot 3^{\circ} \mathrm{C}$.

From the results obtained, values of sonic velocity were calculated from equation (1) and have been plotted in Fig. 1. The experimental points are all within 1 per cent of the curve derived from Yazgan's direct measurements; the standard deviation is 0.7 per cent.

These differences are less than the combined accuracies of the two instruments involved in the comparison, so it seems that equation (1) has been verified over the whole range of pressure from 0.2 to $1.2 \mathrm{kbar}$.

$$
\text { A. T. J. HAYWARD }
$$

National Engineering Laboratory,

East Kilbride,

Glasgow.

Received January 13, 1969.

1 Richards, W. T., Rev. Mod. Phys., 11, 36 (1939).

${ }^{2}$ Rowlinson, J. S., Liquids and Liquid Mixtures (Butterworths, London, 1959).

${ }^{3}$ Hayward, A. T. J., Acta Imeko, 249 (1964).

- Yazgan, E., thesis, Univ. Glasgow (1966).

\section{Effect of Particle Size on the Thermoluminescence of Lithium Fluoride}

Thermoluminescence induced by radiation was one of the earliest ways of detecting ionizing radiation ${ }^{1}$ and has now become widely used as a dosimeter in personal monitoring, radiotherapy and specialized investigations. Because of its high sensitivity, wide dynamic range, lack of fading and relative independence of photon energy ${ }^{2}$, lithium fluoride is one of the materials most frequently used for thermoluminescence dosimetry. Recently Zanelli ${ }^{3}$ has reported that the luminescence efficiency of lithium fluoride varies with the particle size, suggesting that one possible cause of this phenomenon could be the presence of an "inactive" surface layer. This article reports further measurements on the variation in the efficiency of luminescence for small particles with photon energy, and discusses another factor which, in some circumstances, can lead to a decrease in response for small particle sizes. This latter effect and its relevance will be discussed first.

'The energy which is actually deposited in a grain of lithium fluoride depends on the energy balance between the sum of the energies of the electrons leaving the grain and the sum of the energies of the electrons generated within the grain and entering it from the surrounding medium. The total electron energy entering a grain will depend on the mass energy absorption coefficient of the material surrounding the grain (usually air) whereas the total electron energy generated within the grain will depend on the mass energy absorption coefficient of the lithium fluoride. The following considerations apply if 